\title{
Solutions of the $D$-Dimensional Schrödinger Equation with the Hyperbolic Pöschl-Teller Potential plus Modified Ring-Shaped Term
}

\author{
Ibsal A. Assi, ${ }^{1}$ Akpan N. Ikot $\mathbb{D}^{2}{ }^{2}$ and E. O. Chukwuocha ${ }^{2}$ \\ ${ }^{1}$ Department of Physics and Physical Oceanography, Memorial University of Newfoundland, St. John's, NL, Canada A1B $3 X 7$ \\ ${ }^{2}$ Department of Physics, University of Port Harcourt, PMB 5323, Choba, Port Harcourt, Nigeria \\ Correspondence should be addressed to Akpan N. Ikot; ndemikotphysics@gmail.com
}

Received 9 November 2017; Accepted 26 February 2018; Published 22 May 2018

Academic Editor: Shi-Hai Dong

Copyright (c) 2018 Ibsal A. Assi et al. This is an open access article distributed under the Creative Commons Attribution License, which permits unrestricted use, distribution, and reproduction in any medium, provided the original work is properly cited. The publication of this article was funded by SCOAP $^{3}$.

\begin{abstract}
We solve the $D$-dimensional Schrödinger equation with hyperbolic Pöschl-Teller potential plus a generalized ring-shaped potential. After the separation of variable in the hyperspherical coordinate, we used Nikiforov-Uvarov (NU) method to solve the resulting radial equation and obtain explicitly the energy level and the corresponding wave function in closed form. The solutions to the energy eigenvalues and the corresponding wave functions are obtained using the NU method as well.
\end{abstract}

\section{Introduction}

The noncentral potentials in recent times have been an active field of research in physics and quantum chemistry [1-3]. For instance, the occurrence of accidental degeneracy and hidden symmetry in the noncentral potentials and their application in quantum chemistry and nuclear physics are used to describe ring-shaped molecules like benzene and the interaction between deformed pair of nuclei $[4,5]$. It is known that this accidental degeneracy occurring in the ring-shaped potential was explained by constructing an SU (2) algebra [6]. Owing to these applications, many authors have investigated a number of real physical problems on nonspherical oscillator [7], ring-shaped oscillator (RSO) [8], and ring-shaped nonspherical oscillator [9]. Berkdemir [10] had shown that either Coulomb or harmonic oscillator will give a better approximation for understanding the spectroscopy and structure of diatomic molecules in the ground electronic state. Other applications of the ring-shaped potential can be found in ring-shaped organic molecules like cyclic polyenes and benzene [11, 12].

On the other hand, Chen and Dong studied the Schrödinger equation with a new ring-shaped potential [3].
Cheng and Dai investigated modified Kratzer potential plus the new ring-shaped potential using Nikiforov-Uvarov method [13]. Recently, Ikot et al. [14-16] investigated the Schrödinger equation with Hulthen potential plus a new ringshaped potential [3], nonspherical harmonic and Coulomb potential [15], and pseudo-Coulomb potential in the cosmic string space-time [16]. Many authors have used different methods to obtain exact solutions of the wave equation such as the methods of Supersymmetric Quantum Mechanics (SUSY-QM) [17-19], the Tridiagonal Representation Approach (TRA) [20-23], and Nikiforov-Uvarov (NU) method [24-28].

Motivated by the recent studies of the ring-shaped potential [29-32], we proposed a novel hyperbolical Pöschl-Teller potential plus generalized ring-shaped potential of the form (see Figure 1)

$$
\begin{aligned}
V(r, \theta)= & A \tanh ^{2}(\lambda r)+\frac{B}{\tanh ^{2}(\lambda r)} \\
& +\frac{\gamma \cot ^{2} \theta+\zeta \cot \theta \csc \theta+\kappa \csc ^{2} \theta}{r^{2}}
\end{aligned}
$$




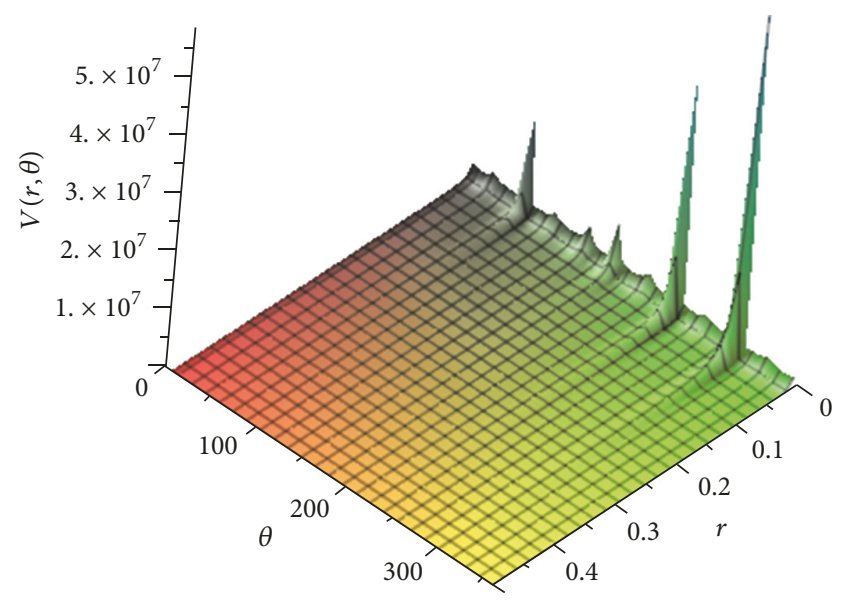

Figure 1: The plot of the novel Pöschl-Teller plus ring-shaped potential as a function of $r$ and $\theta$ for $A=1, B=2, \lambda=0.1, \gamma=2$, $\varsigma=4$, and $\kappa=3$.

where $\lambda$ is the screening parameter and $A, B, \gamma, \zeta$, and $\kappa$ are real potential parameters. As a special case when $\lambda r \rightarrow 0$ with $A \rightarrow m \omega^{2} / 2 \lambda^{2}-\left(\hbar^{2} \alpha / 30 m\right) \lambda^{2}, B \rightarrow\left(\hbar^{2} \alpha / 2 m\right) \lambda^{2}$, and $E \rightarrow$ $E+2 B / 3$; the potential of (1) turns to nonspherical harmonic oscillator plus generalized ring-shaped potential.

$$
\begin{aligned}
V(r, \theta)= & \frac{1}{2} m \omega^{2} r^{2}+\frac{\hbar^{2} \alpha}{2 m r^{2}} \\
& +\frac{\gamma \cot ^{2} \theta+\zeta \cot \theta \csc \theta+\kappa \csc ^{2} \theta}{r^{2}} .
\end{aligned}
$$

\section{D-Dimensional Schrödinger Equation in Hyperspherical Coordinates}

The $D$-dimensional Schrödinger equation is given as follows $[33,34]$ :

$$
\left\{\nabla_{D}^{2}+\frac{2 \mu}{\hbar^{2}}[E-U]\right\} \Psi_{l_{1}, l_{2}, \ldots, l_{D-2}}^{l=l_{D-1}}(\vec{X})=0,
$$

where $\mu$ is the effective mass of two interacting particles, $\hbar$ is Planck's constant, $E$ is the energy eigenvalue, $U$ is the potential energy function, $\vec{X}=\left(r, \theta_{1}, \theta_{2}, \ldots, \theta_{D-1}\right)^{T}$ is the position vector in $D$-dimensions, where $\{\vec{\theta}\}=\left\{\theta_{1}, \theta_{2}, \ldots, \theta_{D-1}\right\}$ is the angular position vector written in terms of hyperspherical coordinates $[35,36]$, and $\nabla_{D}^{2}$ is the $D$-dimensional Laplacian operator given in Appendix B.

The solvable potentials that allow separation of variable in (3) must be of the form

$$
U(\vec{x})=V_{1}(r)+\frac{V_{2}\left(\theta_{D-1}\right)}{r^{2}} .
$$

The separable wave function takes the following form:

$$
\Psi_{l_{1}, l_{2}, \ldots, l_{D-2}}^{l=l_{D-1}}(\vec{X})=r^{-(D-1) / 2} g(r) Y_{l_{1}, l_{2}, \ldots, l_{D-2}}^{l=l_{D-1}}(\vec{\theta}) .
$$

Applying (5) to (3) with the use of (4), we obtain the following radial and angular wave equations:

$$
\begin{aligned}
& {\left[\frac{d^{2}}{d r^{2}}-\frac{(D+2 l-2)^{2}-1}{4 r^{2}}+\frac{2 \mu}{\hbar^{2}}\left[E-V_{1}(r)\right]\right] g(r)} \\
& \quad=0 \\
& {\left[\frac{d^{2}}{d \theta_{j}^{2}}+(j-1) \frac{\cos \theta_{j}}{\sin \theta_{j}} \frac{d}{d \theta_{j}}+\Lambda_{j}-\frac{\Lambda_{j-1}}{\sin ^{2} \theta_{j}}\right] H\left(\theta_{j}\right)} \\
& \quad=0, \\
& {\left[\frac{d^{2}}{d \theta_{D-1}^{2}}+(D-2) \frac{\cos \theta_{D-1}}{\sin \theta_{D-1}} \frac{d}{d \theta_{D-1}}+l(l+D-2)\right.} \\
& \left.\quad-\frac{\Lambda_{D-2}}{\sin ^{2} \theta_{D-1}}+\frac{2 \mu}{\hbar^{2}} V_{2}\left(\theta_{D-1}\right)\right] H\left(\theta_{D-1}\right)=0,
\end{aligned}
$$

where $Y_{l_{1}, l_{2}, \ldots, l_{D-2}}^{l=l_{D_{-1}}}(\vec{\theta})=(1 / \sqrt{2 \pi}) e^{ \pm i m \theta_{1}} \prod_{j=2}^{D-1} H\left(\theta_{j}\right)$, (8) holds for $j \in[2, D-2]$, with $D>3$, and $\Lambda_{j}=l_{j}\left(l_{j}+j-\right.$ 1). Solutions of (8) will not be affected by the presence of the proposed potential and thus are common to different systems and they were done before using different approaches [37]. Consequently, we will only solve (6) and (8) using the Nikiforov-Uvarov method [24, 25].

\section{Nikiforov-Uvarov Method}

Many problems in physics lead to the following second-order linear differential equation [24]:

$$
\left[\frac{d^{2}}{d x^{2}}+\frac{\widetilde{\tau}(x)}{\sigma(x)} \frac{d}{d x}+\frac{\widetilde{\sigma}(x)}{\sigma^{2}(x)}\right] u(x)=0,
$$

where $\sigma(x)$ and $\widetilde{\sigma}(x)$ are polynomials of degree 2 at most and $\widetilde{\tau}(x)$ is at most linear in $x$. Equation (9) is sometimes called of hypergeometric type. Let us consider $u(x)=\phi(x) y(x)$; this will transform (9) to the following differential equation for $y(x)$ :

$$
\left[\frac{d^{2}}{d x^{2}}+\frac{\tau(x)}{\sigma(x)} \frac{d}{d x}+\frac{\bar{\sigma}(x)}{\sigma^{2}(x)}\right] y(x)=0,
$$

where we assumed the following conditions:

$$
\begin{aligned}
\frac{\phi^{\prime}(x)}{\phi(x)}= & \frac{\pi(x)}{\sigma(x)}, \\
\tau(x)= & \tilde{\tau}(x)+2 \pi(x), \\
\bar{\sigma}(x)= & \tilde{\sigma}(x)+\pi^{2}(x)+\pi(x)\left[\widetilde{\tau}(x)-\sigma^{\prime}(x)\right] \\
& +\pi^{\prime}(x) \sigma(x), \\
\pi(x)= & \frac{\sigma^{\prime}(x)-\tilde{\tau}(x)}{2} \\
& \pm \sqrt{\left(\frac{\sigma^{\prime}(x)-\tilde{\tau}(x)}{2}\right)^{2}-\widetilde{\sigma}(x)+k \sigma(x),}
\end{aligned}
$$




$$
k=\eta-\pi^{\prime}(x)
$$

where $k$ and $\eta$ are constants chosen such that $\pi(x)$ is polynomial which is at most linear in $x$ and $\bar{\sigma}(x)=\eta \sigma(x)$. This will transform (10) to the following:

$$
\left[\sigma(x) \frac{d^{2}}{d x^{2}}+\tau(x) \frac{d}{d x}+\eta\right] y(x)=0
$$

where $\sigma(x)$ and $\tau(x)$ are polynomials of degrees 2 and 1 , respectively. In this case, solutions to (12) are polynomials of degree $n ; y(x)=y_{n}\left(x, \eta_{n}\right)$, where $\eta_{n}$ is given as follows:

$$
\eta_{n}=-n \tau^{\prime}-\frac{1}{2} n(n-1) \sigma^{\prime \prime}
$$

Equation (13) will be used to obtain the energy spectrum formula of the quantum mechanical system. We should point out here that the polynomial solutions to (12) for $\tau^{\prime}<0$ and $\tau=0$ on the boundaries of the finite space (the latter case is omitted for infinite space) are the classical orthogonal polynomials. It is well known that each set of polynomials is associated with a weight function $\rho(x)$. For the polynomial solutions to (12), this function must be bounded on the domain of the system and must satisfy $(\sigma \rho)^{\prime}=\tau \rho$. This weight function will be used to construct the Rodrigues formula for these polynomials, which reads

$$
y_{n}(x)=\frac{B_{n}}{\rho(x)} \frac{d^{n}}{d x^{n}}\left[\sigma^{n}(x) \rho(x)\right],
$$

where $B_{n}$ is just a constant obtained by the normalization conditions and $n=0,1,2, \ldots$.

\section{The Solutions of the $D$-Dimensional Radial Equation}

We use the NU method to solve (6); in the presence of our potential,

$$
\begin{aligned}
& \frac{d^{2} g(r)}{d r^{2}} \\
& +\frac{2 \mu}{\hbar^{2}}\left[E-A \tanh ^{2}(\lambda r)-\frac{B}{\tanh ^{2}(\lambda r)}-\frac{\gamma_{D} \hbar^{2}}{2 \mu} \frac{1}{r^{2}}\right] \\
& \cdot g(r)=0,
\end{aligned}
$$

where $\gamma_{D}=\left((D+2 l-2)^{2}-1\right) / 4$. Equation (15) cannot be solved analytically due to the centrifugal term $1 / r^{2}$. Different authors used different approximation techniques to allow an approximate analytical solution of (15) and these methods rely on Taylor expansion of the centrifugal potential in terms of the other components of the potential of interest [38]. In this work, we use the following approximation obtained by Taylor expansion [39]:

$$
\frac{1}{\lambda^{2} r^{2}} \approx-\frac{2}{3}-\frac{1}{3} \tanh ^{2}(\lambda r)+\frac{1}{\tanh ^{2}(\lambda r)} .
$$

The advantage of this approximation is that it is valid not only for $\lambda r \ll 1$ but also for $0 \leq \lambda r \leq 2$ with high accuracy. Also, it satisfies the limits on $1 / r^{2}$ at zero and infinity; that is, $\lim _{r \rightarrow 0} \mathrm{RHS}=1 / \lambda^{2} r^{2}$ and $\lim _{r \rightarrow \infty} \mathrm{RHS}=0$, where RHS denotes the right-hand side of (16). Now, Using (16) back in (15), we get

$$
\left[\frac{d^{2}}{d r^{2}}-4 \lambda^{2} \widetilde{A} \tanh ^{2} \lambda r-\frac{4 \lambda^{2} \widetilde{B}}{\tanh ^{2} \lambda r}+4 \lambda^{2} \widetilde{E}\right] g(r)=0,
$$

where $4 \lambda^{2} \widetilde{E}=\left(2 \mu / \hbar^{2}\right)\left[E+\gamma_{D} \hbar^{2} \lambda^{2} / 3 \mu\right], 4 \lambda^{2} \widetilde{A}=\left(2 \mu / \hbar^{2}\right)(A-$ $\left.\gamma_{D} \hbar^{2} \lambda^{2} / 6 \mu\right)$, and $4 \lambda^{2} \widetilde{B}=\left(2 \mu / \hbar^{2}\right)\left[B+\gamma_{D} \hbar^{2} \lambda^{2} / 2 \mu\right]$. Making change of variable $s=\tanh ^{2} \lambda r$ and by writing $g(s)=$ $\phi(s) y(s)$, this transforms (17) to (9) with the polynomials being $\sigma=s-s^{2}, \widetilde{\tau}=-(3 s-1) / 2$, and $\widetilde{\sigma}=\widetilde{E} s-\widetilde{A} s^{2}-\widetilde{B}$. We now use (11d) to calculate $\pi(s)$, which reads

$$
\begin{aligned}
\pi(s)= & \frac{1-s}{4} \\
& \pm \sqrt{\left(\frac{1-s}{4}\right)^{2}+\widetilde{A} s^{2}-\widetilde{E} s+\widetilde{B}+k\left(s-s^{2}\right) .}
\end{aligned}
$$

The choice of $k$ that makes (18) a polynomial of first degree must satisfy $c_{1}^{2}=c_{2} c_{3}$, where $c_{1}=16 k-8-16 \widetilde{E}, c_{2}=1+$ $16 \widetilde{A}-16 k$, and $c_{3}=4(1+16 \widetilde{B})$. This gives

$$
\pi(s)=\frac{1-s}{4} \pm \frac{1}{4} \sqrt{c_{2}}\left(s+\frac{c_{1}}{2 c_{2}}\right),
$$

where we will pick the negative part in (19) which makes $\tau^{\prime}<$ 0 . The function $\tau(s)$ can be easily calculated using (11b):

$$
\tau(s)=1-2 s-\frac{1}{2} \sqrt{c_{2}}\left(s+\frac{c_{1}}{2 c_{2}}\right) .
$$

Using (19) and (20) in (13) and (11e), we get

$$
\left[\frac{4 k}{(2 n+1)}-(2 n+1)\right]^{2}=(1+16 \widetilde{A}-16 k) .
$$

Solutions of (21) for $k$ are

$$
4 k=-(2 n+1)^{2} \pm(2 n+1) \sqrt{1+16 \widetilde{A}} .
$$

The next step is to use the value of $c_{2}$ in (21) with the constraint on $k$ mentioned in (18), which is $c_{1}^{2}=c_{2} c_{3}$; we obtain

$$
[8+16 \widetilde{E}-16 k]^{2}=4(1+16 \widetilde{B})(1+16 \widetilde{A}-16 k) .
$$

The conditions for bound states are $k \leq 0$ and $\widetilde{A}, \widetilde{B} \geq-1 / 16$. Using (22) in (23), we write the bound states formula as follows:

$$
\begin{aligned}
\widetilde{E}_{n l}^{D} & =-\frac{1}{4}(2 n+1)^{2}-\frac{1}{4}(2 n+1) \sqrt{1+16 \widetilde{A}}-\frac{1}{2} \pm \frac{1}{8} \\
& \cdot \sqrt{(1+16 \widetilde{B})\left(1+16 \widetilde{A}+4(2 n+1)^{2}+4(2 n+1) \sqrt{1+16 \widetilde{A}}\right)} .
\end{aligned}
$$

In terms of the original parameters $A, B$, and $E$, the spectrum formula in $D$-dimensions reads 


$$
\begin{aligned}
\frac{2 \mu}{\hbar^{2}} E_{n l}^{D}= & -\frac{2 \gamma_{D} \lambda^{2}}{3}-\lambda^{2}(2 n+1)^{2}-\lambda^{2}(2 n+1) \sqrt{1+\frac{8 \mu}{\lambda^{2} \hbar^{2}}\left(A-\frac{\gamma_{D} \hbar^{2} \lambda^{2}}{6 \mu}\right)}-2 \lambda^{2} \pm \frac{\lambda^{2}}{2} \sqrt{\left(1+\frac{8 \mu}{\lambda^{2} \hbar^{2}}\left[B+\frac{\gamma_{D} \hbar^{2} \lambda^{2}}{2 \mu}\right]\right)} \\
& \times \sqrt{1+\frac{8 \mu}{\lambda^{2} \hbar^{2}}\left(A-\frac{\gamma_{D} \hbar^{2} \lambda^{2}}{6 \mu}\right)+4(2 n+1)^{2}+4(2 n+1) \sqrt{1+\frac{8 \mu}{\lambda^{2} \hbar^{2}}\left(A-\frac{\gamma_{D} \hbar^{2} \lambda^{2}}{6 \mu}\right)}},
\end{aligned}
$$

where conditions for bound states become $A \geq\left(\lambda^{2} \hbar^{2} /\right.$ $2 \mu)\left(\gamma_{D} / 3-1 / 4\right)$ and $B \geq-\left(\lambda^{2} \hbar^{2} / 2 \mu\right)\left(\gamma_{D}+1 / 4\right)$. We will only take the (-) sign in (25) as explained below. The $s$ wave spectrum formula in three dimensions is the only exact solution that is obtained by setting $\gamma_{D}=0$ in (25). However, for other higher states, the above solution is acceptable with high accuracy as far as the condition $0 \leq \lambda r \leq 2$ is satisfied.

The transformation $s=\tanh ^{2} \lambda x$ makes the domain of the function be $[0,1]$. This suggests a change of variable $z=2 s-1$ to bring the domain to that of Jacobi polynomials which are well-known classical orthogonal polynomials. By using (18) in (11a), we obtain $\phi(z)=2^{c_{4}-2 c_{5}}(1+z)^{c_{5}}(1-z)^{c_{5}-c_{4}}$, where $4 c_{4}=1+\sqrt{c_{2}}$ and $8 c_{5}=2-c_{1} / \sqrt{c_{2}}$. The weight function can be easily calculated using (20) in $(\sigma \rho)^{\prime}=\tau \rho$, which gives $\rho(z)=2^{2 c_{7}+c_{6}}(1+z)^{-c_{7}}(1-z)^{-c_{6}-c_{7}}$, where $2 c_{6}=8+\sqrt{c_{2}}$ and $4 c_{7}=c_{1} / \sqrt{c_{2}}$. The solution of (12) in our case is written in the following Rodrigues formula:

$$
\begin{aligned}
y_{n}(z) & =C_{n}(1+z)^{c_{7}} \\
& \cdot(1-z)^{c_{6}+c_{7}} \frac{d^{n}}{d z^{n}}\left[(1+z)^{-c_{7}+n}(1-z)^{n-c_{6}-c_{7}}\right] .
\end{aligned}
$$

By comparison to Jacobi polynomials, we conclude that $y_{n}(z)=P_{n}^{\left(-c_{6}-c_{7},-c_{7}\right)}(z)$, where $P_{n}^{\left(-c_{6}-c_{7},-c_{7}\right)}(z)$ is the Jacobi polynomial of order $n$ in $z$. Thus, the bound state solution of the radial wave equation now reads

$$
\begin{aligned}
g_{n}(r)= & \Omega_{n}(\tanh \lambda r)^{-c_{6}-c_{7}+1 / 2}(\operatorname{sech} \lambda r)^{-c_{7}} \\
& \cdot P_{n}^{\left(-c_{6}-c_{7},-c_{7}\right)}\left[2 \tanh (\lambda r)^{2}-1\right],
\end{aligned}
$$

where $\Omega_{n}$ is just a normalization constant. We must clarify here that, for Jacobi polynomials, we have to have $\left(-c_{6}-\right.$ $\left.c_{7}\right),-c_{7}>-1$. Thus, the parameters $c_{1}$ and $c_{2}$ are chosen to satisfy $c_{1}<4 \sqrt{c_{2}}$ and $2 c_{2}+c_{1}<-12 \sqrt{c_{2}}$. Moreover, since those parameters depend on the energy as we mentioned previously, this yields us to reject the (+) sign in (24) and (25). Consequently, bound states occur for $\widetilde{B}>35 / 16$ (which does not violate the old restriction $\widetilde{B} \geq-1 / 16)$ and $|\widetilde{E}|>|k-1 / 2|$. The latter condition on $E$ is already satisfied as we can see in (25), so we do not have to worry about it.

To calculate the normalization constant $\Omega_{n}$, we first use the following identity of Jacobi polynomials [40]:

$$
\begin{aligned}
& P_{n}^{(a, b)}(y) \\
& \quad=\frac{1}{2^{n}} \sum_{m=0}^{n}\left(\begin{array}{c}
n+a \\
m
\end{array}\right)\left(\begin{array}{c}
n+b \\
n-m
\end{array}\right)(1-y)^{m-n}(1+y)^{m} .
\end{aligned}
$$

Next, we use the normalization constraint $\int_{0}^{\infty}|g(r)|^{2} d r=$ $\int_{-1}^{+1}|g(y)|^{2}(d y / \sqrt{2} \lambda \sqrt{1+y}(1-y))=1$, where $y=$ $2 \tanh ^{2}(\lambda r)-1$; this gives

$$
\begin{aligned}
& \left(\left|\Omega_{n}\right|^{2} / \lambda 2^{n+1 / 2}\right) \sum_{m=0}^{n}\left(\begin{array}{c}
n+a \\
m
\end{array}\right)\left(\begin{array}{c}
n+b \\
n-m
\end{array}\right) \\
& \quad \cdot \int_{-1}^{+1}(1-y)^{2 a+m-n-1}(1+y)^{b+m} P_{n}^{(a, b)}(y) d y=1 .
\end{aligned}
$$

To calculate the integral in (29), we will use the following very useful integral formula [41]:

$$
\begin{aligned}
& \int_{-1}^{+1}(1-y)^{c}(1+y)^{d} P_{n}^{(a, b)}(y) d y \\
& =\frac{2^{c+d+1} \Gamma(c+1) \Gamma(d+1) \Gamma(n+a+1)}{\Gamma(n+1) \Gamma(c+d+2) \Gamma(a+1)} \\
& \quad \times{ }_{3} F_{2}(-n, n+a+b+1, c+1 ; a+1, c+d+2 ; 1),
\end{aligned}
$$

where ${ }_{3} F_{2}(a, b, c ; d, e ; f)$ is the generalized hypergeometric function [41]. By direct comparison between (29) and (30) we get $\Omega_{n}=1 / \sqrt{\Lambda_{n}}$, where $\Lambda_{n}$ is given as follows:

$$
\begin{aligned}
\Lambda_{n} & =\frac{1}{\lambda 2^{n+1 / 2}} \sum_{m=0}^{n}\left(\begin{array}{c}
n+a \\
m
\end{array}\right)\left(\begin{array}{c}
n+b \\
n-m
\end{array}\right) \\
& \cdot \frac{2^{2 a+2 m-n+b} \Gamma(2 a+m-n) \Gamma(b+m+1) \Gamma(n+a+1)}{\Gamma(n+1) \Gamma(2 a+2 m-n+b+1) \Gamma(a+1)} \\
& \times{ }_{3} F_{2}(-n, n+a+b+1,2 a+m-n ; a+1,2 a+2 m \\
& -n+b+1 ; 1),
\end{aligned}
$$

where $a=-c_{6}-c_{7}$ and $b=-c_{7}$.

The only issue that is left for discussion in this section is that the solutions of the radial wave equation $\left\{g_{n}(r)\right\}_{n=0}^{N}$, where $N$ denotes the maximum number in which we get bound states, are not orthogonal! But they are normalized as we discussed above. We know that Hermitian operators with distinct eigenvalues must have orthogonal eigenvectors [42]. To solve this problem, one must use the Gram-Schmidt (GS) method to obtain an orthonormal set $\left\{\phi_{n}(r)\right\}_{n=0}^{N}$ by linear combinations [43]. The latter set will be the solutions of the radial wave equation. The process is a bit lengthy and we will not be able to do it here. However, we encourage the interested reader to do these calculations by referring to the process of GS. 


\section{Solutions of the Angular Equations}

It is well known from literature that solutions of (7) are written in terms of Jacobi polynomials as follows [41]:

$$
H(y)=N_{n}(1-y)^{\alpha}(1+y)^{\beta} P_{n}^{(c, d)}(y),
$$

where $N_{n}$ is just a constant factor, $2 \beta+j / 2=d+1$, and $2 \alpha+$ $j / 2=c+1$. Moreover, the latter parameters are written in terms of the quantum numbers as $c=d=c_{j}=l_{j-1}+(j-2) / 2$, which yields $\alpha=\beta=l_{j-1} / 2$ and $n=l_{j}-l_{j-1}$. The above solution was obtained using different methods including the NU technique [24]. Hence, solutions of (7) are written below:

$$
H\left(\theta_{j}\right)=N_{n}\left(\sin \theta_{j}\right)^{l_{j-1}} P_{n}^{\left(c_{j}, c_{j}\right)}\left(\cos \theta_{j}\right) .
$$

To solve (8), we introduce coordinate transformation as $y=$ $\cos \theta_{D-1}$, which gives

$$
\begin{gathered}
{\left[\left(1-y^{2}\right) \frac{d^{2}}{d y^{2}}-(D-1) y \frac{d}{d y}+l(l+D-2)\right.} \\
\left.-\frac{\Lambda_{D-2}}{1-y^{2}}+U(y)\right] H(y)=0,
\end{gathered}
$$

where $U(y)=\left(2 \mu / \hbar^{2}\right) V_{2}(y)=\left(\gamma^{\prime} y^{2}+\zeta^{\prime} y+\kappa^{\prime}\right) /\left(1-y^{2}\right)$ for real parameters $\left\{\gamma^{\prime}, \zeta^{\prime}, \kappa^{\prime}\right\}$ are related to $\{\gamma, \zeta, \kappa\}$ by a factor of $2 \mu / \hbar^{2}$. Equation (34) is of hypergeometric type with the polynomials being $\sigma(y)=1-y^{2}, \widetilde{\tau}(y)=-(D-1) y$, and $\widetilde{\sigma}(y)=\eta_{2} y^{2}+\eta_{1} y+\eta_{0}$, where $\eta_{2}=\gamma^{\prime}-l(l+D-2), \eta_{1}=\zeta^{\prime}$, and $\eta_{0}=\kappa^{\prime}+l(l+D-2)-\Lambda_{D-2}$. The solutions of (34) are written as $H(y)=\phi(y) Y(y)$, where $\phi(y)$ satisfies (11a). Next, we need to find the function $\pi(y)$ using (11d); we find that this function takes the following form:

$$
\pi(y)=\frac{\left(D-3-2 u_{0}\right) y}{2}+\frac{\eta_{1}}{2 u_{0}},
$$

where $u_{0}=\sqrt{((D-3) / 2)^{2}-\eta_{2}-k}$ and the parameter $k$ defined in (11e) must satisfy $4 k-4 \eta_{0}=\eta_{1}^{2} / u_{0}^{2}$. The latter constraint will be used later to obtain the eigenvalues of (34). Now, we use (35) in (11b); we get $\tau(y)=-2\left(1+u_{0}\right) y+\eta_{1} / u_{0}$, which satisfies $d \tau(y) / d y<0$. Using (11a), we can obtain $\phi(y)$ to be $\phi(y)=(1-y)^{-\left(u_{1}+u_{2}\right) / 2}(1+y)^{\left(u_{2}-u_{1}\right) / 2}$, where $2 u_{1}=\left(D-3-2 u_{0}\right)$ and $\eta_{1}=2 u_{0} u_{2}$. We can also calculate the weight function by solving $(\sigma \rho)^{\prime}=\tau \rho$, which gives $\rho(y)=(1-y)^{u_{0}-u_{2}}(1+y)^{u_{0}+u_{2}}$. The Rodrigues formula of the polynomials $Y(y)$ reads

$$
\begin{aligned}
Y_{n}(y)= & \frac{\xi_{n}}{(1-y)^{u_{0}-u_{2}}(1+y)^{u_{0}+u_{2}}} \\
& \cdot \frac{d^{n}}{d y^{n}}\left[(1-y)^{n+u_{0}-u_{2}}(1+y)^{n+u_{0}+u_{2}}\right],
\end{aligned}
$$

where $\xi_{n}$ is just a constant. By direct comparison with the Rodrigues formula of Jacobi polynomials [xx], we conclude that $Y_{n}(y)=P_{n}^{\left(u_{0}-u_{2}, u_{0}+u_{2}\right)}(y)$. As required by Jacobi polynomials, we must impose that $u_{0} \pm u_{2}>-1$. Now, we use (35) and (13) in (11e) to obtain the following quadratic formula for $k$ :

$$
\begin{aligned}
& {\left[k-n^{2}-n+\frac{(D-3)}{2}\right]^{2}} \\
& \quad=(2 n+1)^{2}\left[\left(\frac{D-3}{2}\right)^{2}-\eta_{2}-k\right] .
\end{aligned}
$$

The solutions of (37) are given below:

$$
\begin{aligned}
k= & \frac{1}{2}\left[2-D-2 n-2 n^{2}\right. \\
& \left. \pm(1+2 n) \sqrt{(D-2)^{2}-4 \eta_{2}}\right] .
\end{aligned}
$$

Moreover, we use $4 k-4 \eta_{0}=\eta_{1}^{2} /\left(((D-3) / 2)^{2}-\eta_{2}-k\right)$ to obtain another solution for $k$ :

$$
\begin{aligned}
8 k & =9+D^{2}-6 D+4 \eta_{0}-4 \eta_{2} \\
& \pm \sqrt{\left((D-3)^{2}+4 \eta_{0}-4 \eta_{2}\right)^{2}-16\left(\eta_{1}^{2}+\eta_{0}\left((D-3)^{2}-4 \eta_{2}\right)\right)} .
\end{aligned}
$$

Direct comparison between (38) and (39) gives

$$
\begin{gathered}
(2 n+1)^{2}=-\frac{(D-1)^{2}+4 \eta_{0}-4 \eta_{2}-2}{2}, \\
(2 n+1)^{2}\left[(D-2)^{2}-4 \eta_{2}\right] \\
=\frac{\left((D-3)^{2}+4 \eta_{0}-4 \eta_{2}\right)^{2}}{16} \\
-\left(\eta_{1}^{2}+\eta_{0}\left((D-3)^{2}-4 \eta_{2}\right)\right) .
\end{gathered}
$$

In the next section, we will consider different examples and try to obtain the unknown parameters for each case.

\section{Results and Discussions}

In this section, we will discuss different examples that are considered as special cases of the potential in (1).

As a first example, we consider the case when $\gamma^{\prime}=\zeta^{\prime}=0$ and $\kappa^{\prime} \neq 0$, which is equivalent to the following noncentral hyperbolic potential:

$$
V(r, \theta)=A \tanh ^{2}(\lambda r)+\frac{B}{\tanh ^{2}(\lambda r)}+\frac{\kappa \csc ^{2} \theta}{r^{2}}
$$

In this case, we have $u_{2}=0$. Thus, solution of (34) reads

$$
H_{n}\left(\theta_{D-1}\right)=N_{n}\left(\sin \theta_{D-1}\right)^{-u_{1}} P_{n}^{\left(u_{0}, u_{0}\right)}\left(\cos \theta_{D-1}\right),
$$


where $2 u_{1}=\left(D-3-2 u_{0}\right), u_{0}=$ $\sqrt{((D-3) / 2)^{2}+l(l+D-2)-k}$, and $k$ is given below:

$$
8 k=9+D^{2}-6 D+4 \eta_{0}+4 l(l+D-2) \pm \sqrt{\left((D-3)^{2}+4 \eta_{0}+4 l(l+D-2)\right)^{2}-16\left(\eta_{0}\left((D-3)^{2}+4 l(l+D-2)\right)\right)}
$$

and the corresponding eigenvalue is obtain from (41) as

$$
\begin{aligned}
(2 n+1)^{2}\left[(D-2)^{2}+4 l(l+D-2)\right] \\
=\frac{\left((D-3)^{2}+4 \eta_{0}+4 l(l+D-2)\right)^{2}}{16} \\
-\left(\eta_{0}\left((D-3)^{2}+4 l(l+D-2)\right)\right) .
\end{aligned}
$$

(2) The next special case of our potential model is considered when we choose the ring-shaped parameters $\gamma= \pm \varsigma$ and $\kappa \neq$ 0 , which corresponds to the following potential:

$$
\begin{aligned}
V(r, \theta)= & A \tanh ^{2}(\lambda r)+\frac{B}{\tanh ^{2}(\lambda r)} \\
& +\frac{\gamma\left(\cot ^{2} \theta \pm \cot \theta \csc \theta\right)+\kappa \csc ^{2} \theta}{r^{2}} .
\end{aligned}
$$

$8 k$

$$
\begin{aligned}
& =9+D^{2}-6 D+4 \eta_{0} \mp 4 \varsigma+4 l(l+D-2) \\
& \pm \sqrt{\left((D-3)^{2}+4 \eta_{0} \mp 4 \varsigma+4 l(l+D-2)\right)^{2}-16\left(\varsigma^{2}+\eta_{0}\left((D-3)^{2} \mp 4 \varsigma+4 l(l+D-2)\right)\right)}, \\
(2 n+1)^{2}\left[(D-2)^{2} \mp 4 \varsigma+4 l(l+D-2)\right] & \\
= & \frac{\left((D-3)^{2}+4 \eta_{0} \mp 4 \varsigma+4 l(l+D-2)\right)^{2}}{16}-\left(\varsigma^{2}+\eta_{0}\left((D-3)^{2} \mp 4 \varsigma+4 l(l+D-2)\right)\right) .
\end{aligned}
$$

The associated nonnormalized wave function is obtained as

$$
\begin{aligned}
H_{n}\left(\theta_{D-1}\right)= & N_{n}\left(1-\cos \theta_{D-1}\right)^{-\left(u_{1}+u_{2}\right) / 2} \\
& \cdot\left(1+\cos \theta_{D-1}\right)^{\left(u_{2}-u_{1}\right) / 2} \\
& \cdot P_{n}^{\left(u_{0}-u_{2}, u_{0}+u_{2}\right)}\left(\cos \theta_{D-1}\right)
\end{aligned}
$$

(3) Another special case of our study is when $\varsigma=0$ and $\gamma$, $\kappa \neq 0$, which corresponds to the following potential:

$$
\begin{aligned}
V(r, \theta)= & A \tanh ^{2}(\lambda r)+\frac{B}{\tanh ^{2}(\lambda r)} \\
& +\frac{(\gamma+\kappa) \cot ^{2} \theta+\kappa}{r^{2}} .
\end{aligned}
$$

Under these conditions, we have

$$
\begin{aligned}
& \eta_{1}=\varsigma, \\
& \eta_{2}= \pm \varsigma-l(l+D-2), \\
& u_{1}=\frac{\left(D-3-2 u_{0}\right)}{2}, \\
& u_{2}=\frac{\varsigma}{2 u_{0}}, \\
& u_{0}=\sqrt{\left(\frac{D-3}{2}\right)^{2} \mp(\varsigma-l(l+D-2))-k .}
\end{aligned}
$$

The $k$ values and the corresponding eigenvalues are obtained as follows: 
Under this special case, we obtain the $k$ parameter, the eigenvalues, and the corresponding wave function as follows:

$$
\begin{aligned}
& 8 k=9+D^{2}-6 D+4\left(\kappa+l(l+D-2)-\Lambda_{D-2}\right)-4(\gamma-l(l+D-2)) \\
& \quad \pm \sqrt{\left((D-3)^{2}+4\left(\kappa+l(l+D-2)-\Lambda_{D-2}\right)-4(\gamma-l(l+D-2))\right)^{2}-16\left(\eta_{0}\left((D-3)^{2}-4 \eta_{2}\right)\right)} \\
& (2 n+1)^{2}\left[(D-2)^{2}-4(\gamma-l(l+D-2))\right]=\frac{\left((D-3)^{2}+4\left(\kappa+l(l+D-2)-\Lambda_{D-2}\right)-4(\gamma-l(l+D-2))\right)^{2}}{16} \\
& \quad-\left(\left(\kappa+l(l+D-2)-\Lambda_{D-2}\right)\left((D-3)^{2}-4(\gamma-l(l+D-2))\right)\right) \\
& H_{n}\left(\theta_{D-1}\right)=N_{n}\left(\sin \theta_{D-1}\right)^{-u_{1}} P_{n}^{\left(u_{0}, u_{0}\right)}\left(\cos \theta_{D-1}\right) .
\end{aligned}
$$

However, one needs to be careful here as, for $\gamma=-\kappa$, there will be no ring-shaped term and one ends up with hyperbolic PT potential plus pseudo centrifugal term:

$$
V(r, \theta)=A \tanh ^{2}(\lambda r)+\frac{B}{\tanh ^{2}(\lambda r)}+\frac{\kappa}{r^{2}} .
$$

(4) We consider the last special case for $\gamma=0$ and $\kappa= \pm \varsigma$, which corresponds to the potential of the form

$$
\begin{aligned}
V(r, \theta)= & A \tanh ^{2}(\lambda r)+\frac{B}{\tanh ^{2}(\lambda r)} \\
& +\frac{\varsigma\left[\cot \theta \csc \theta \pm \csc ^{2} \theta\right]}{r^{2}} .
\end{aligned}
$$

The following parameters are obtained under this case:

$$
\eta_{1}= \pm \varsigma
$$

$$
\begin{aligned}
& \eta_{2}=-l(l+D-2), \\
& \eta_{0}= \pm \varsigma+l(l+D-2)-\Lambda_{D-2} \\
& u_{1}=\left(\frac{D-3-2 u_{0}}{2}\right),
\end{aligned}
$$

$$
\begin{aligned}
& u_{0}=\sqrt{\left(\frac{D-3}{2}\right)^{2}+l(l+D-2)-k}, \\
& u_{2}=\frac{ \pm \varsigma}{2 u_{0}} .
\end{aligned}
$$

Using (55), we obtain the $k$ parameter, the eigenvalues, and the corresponding wave function for this special case as follows:

$$
\begin{aligned}
& 8 k=9+D^{2}-6 D+4\left( \pm \varsigma+l(l+D-2)-\Lambda_{D-2}\right)+4 l(l+D-2) \\
& \pm \sqrt{\left((D-3)^{2}+4\left( \pm \varsigma+l(l+D-2)-\Lambda_{D-2}\right)+4 l(l+D-2)\right)^{2}-16\left(( \pm \varsigma)^{2}+\left( \pm \varsigma+l(l+D-2)-\Lambda_{D-2}\right)\left((D-3)^{2}+4 l(l+D-2)\right)\right)} \\
& (2 n+1)^{2}\left[(D-2)^{2}+4 l(l+D-2)\right]=\frac{\left((D-3)^{2}+4\left( \pm \varsigma+l(l+D-2)-\Lambda_{D-2}\right)+4 l(l+D-2)\right)^{2}}{16} \\
& \quad-\left(( \pm \varsigma)^{2}+\left( \pm \varsigma+l(l+D-2)-\Lambda_{D-2}\right)\left((D-3)^{2}+4 l(l+D-2)\right)\right), \\
& H_{n}\left(\theta_{D-1}\right)=N_{n}\left(1-\cos \theta_{D-1}\right)^{-\left(u_{1}+u_{2}\right) / 2}\left(1+\cos \theta_{D-1}\right)^{\left(u_{2}-u_{1}\right) / 2} P_{n}^{\left(u_{0}-u_{2}, u_{0}+u_{2}\right)}\left(\cos \theta_{D-1}\right) .
\end{aligned}
$$

\section{Conclusions}

In this paper, we have obtained analytically the solutions of the $D$-dimensional Schrödinger potential with hyperbolic
Pöschl-Teller potential plus a generalized ring-shaped term. We employed NU and trial function methods to solve the radial and angular parts of the Schrödinger equation, respectively. This result is new and has never been reported in the 
available literature to the best of our knowledge. Finally, this result can find many applications in atomic and molecular physics and thermodynamic properties [43].

\section{Appendix}

\section{A. Jacobi Polynomials}

Jacobi polynomials $P_{n}^{(\mu, \nu)}(y)$ defined on $[-1,1]$ are solutions of the following second-order linear differential equation [8]:

$$
\begin{aligned}
& \left\{\left(1-y^{2}\right) \frac{d^{2}}{d y^{2}}-[(\mu+\nu+2) y+\mu-\nu] \frac{d}{d y}\right. \\
& +n(n+\mu+v+1)\} P_{n}^{(\mu, \nu)}(y)=0 .
\end{aligned}
$$

We also mention their orthogonality relation:

$$
\begin{aligned}
& \int_{-1}^{1}(1-y)^{\mu}(1+y)^{\nu} P_{n}^{(\mu, \nu)} P_{m}^{(\mu, \nu)} d y \\
& \quad=\frac{2^{\mu+\nu+1}}{2 n+\mu+\nu+1} \frac{\Gamma(n+\mu+1) \Gamma(n+\nu+1)}{\Gamma(n+\mu+\nu+1) n !} \delta_{n, m} .
\end{aligned}
$$

\section{B. Hyperspherical Coordinates}

The $D$-dimensional position vector $\vec{x}=\left(r, \theta_{1}, \ldots, \theta_{D-1}\right)$ is defined in terms of hyperspherical Cartesian coordinates below [36]:

$$
\begin{aligned}
& x_{1}=r \cos \theta_{1} \sin \theta_{2} \cdots \sin \theta_{D-1}, \\
& x_{2}=r \sin \theta_{1} \sin \theta_{2} \cdots \sin \theta_{D-1}, \\
& x_{j}=r \cos \theta_{j-1} \sin \theta_{j} \cdots \sin \theta_{D-1},
\end{aligned}
$$

where $j=3,4, \ldots, D-1, x_{D}=r \cos \theta_{D-1}$, and $\sum_{j=1}^{D} x_{j}^{2}=r^{2}$. For $D=2$, this is the case of polar coordinates $(r, \varphi)$ with $x_{1}=x=r \cos \varphi$ and $x_{2}=y=r \sin \varphi$, whereas $D=3$ represents the spherical coordinates $(r, \varphi, \theta)$, where $x_{1}=x=$ $r \cos \varphi \sin \theta, x_{2}=y=r \cos \varphi \sin \theta$, and $x_{3}=z=r \cos \theta$.

The volume element in $D$-dimension is defined as $d V=$ $r^{D-1} d r \prod_{j=1}^{D-1}\left(\sin \theta_{j}\right)^{j-1} d \theta_{j}$, where $r \in\left[0, \infty\left[, \theta_{1} \in[0,2 \pi]\right.\right.$, and $\theta_{j} \in[0, \pi]$ for $j \geq 2$. The Laplacian operator in $D$ dimensions is defined below:

$$
\begin{aligned}
\nabla_{D}^{2} & =\frac{\partial^{2}}{\partial r^{2}}+\frac{D-1}{r} \frac{\partial}{\partial r}+\frac{1}{r^{2}} \\
& \times\left[\frac{1}{\sin ^{D-2} \theta_{D-1}} \frac{\partial}{\partial \theta_{D-1}}\left(\sin ^{D-2} \theta_{D-1} \frac{\partial}{\partial \theta_{D-1}}\right)\right. \\
& \left.-\frac{L_{D-2}^{2}}{\sin ^{2} \theta_{D-1}}\right] .
\end{aligned}
$$

Finally, we mention the normalization conditions of the wave function in $D$-dimensions:

$$
\int_{0}^{\infty}\left|g_{n}(r)\right|^{2} d r=1,
$$

$$
\prod_{j=2}^{D-1} \int_{0}^{\pi}\left|H\left(\theta_{j}\right)\right|^{2}\left(\sin \theta_{j}\right)^{j-1} d \theta_{j}=1 .
$$

\section{Conflicts of Interest}

The authors declare that they have no conflicts of interest.

\section{References}

[1] M. C. Zhang, G. H. Sun, and S.-H. Dong, "Exactly complete solutions of the Schrödinger equation with a spherically harmonic oscillatory ring-shaped potential," Physics Letters A, vol. 374, no. 5, pp. 704-708, 2010.

[2] A. D. Alhaidari, "Scattering and bound states for a class of non-central potentials," Journal of Physics A: Mathematical and General, vol. 38, no. 15, pp. 3409-3429, 2005.

[3] C.-Y. Chen and S.-H. Dong, "Exactly complete solutions of the Coulomb potential plus a new ring-shaped potential," Physics Letters A, vol. 335, no. 5-6, pp. 374-382, 2005.

[4] C. Berkdemir and R. Sever, "Modified $\ell$-states of diatomic molecules subject to central potentials plus an angle-dependent potential," Journal of Mathematical Chemistry, vol. 46, no. 4, pp. 1122-1136, 2009.

[5] H. Hartmann and D. Schuch, "Spin-orbit coupling for the motion of a particle in a ring-shaped potential," International Journal of Quantum Chemistry, vol. 18, pp. 125-141, 1980.

[6] G. E. Draganascu, C. Campigotto, and M. Kibler, "On a generalized Aharonov-Bohm plus Coulomb system," Physics Letters A, vol. 170, no. 5, pp. 339-343, 1992.

[7] C.-Y. Chen, D.-S. Sun, and F.-L. Lu, "The relativistic bound states of the Hartmann potentials," Physica Scripta, vol. 74, no. 4, pp. 405-409, 2006.

[8] C. Quesne, "An sl(4,R) Lie algebraic treatment of the first family of Poschl-Teller potentials," Journal of Physics A: Mathematical and General, vol. 21, no. 24, pp. 4487-4500, 1988.

[9] S.-H. Dong, G.-H. Sun, and M. Lozada-Cassou, "An algebraic approach to the ring-shaped non-spherical oscillator," Physics Letters A, vol. 328, no. 4-5, pp. 299-305, 2004.

[10] C. Berkdemir, "A novel angle-dependent potential and its exact solution," Journal of Mathematical Chemistry, vol. 46, no. 1, pp. 139-154, 2009.

[11] H. Hassanabadi, A. N. Ikot, and S. Zarrinkamar, "Exact solution of Klein-Gordon with the Pöschl-Teller double-ring-shaped Coulomb potential," Acta Physica Polonica A, vol. 126, no. 3, pp. 647-651, 2014.

[12] M.-C. Zhang, B. An, and H.-F. Guo-Qing, "Exact solutions of a new Coulomb ring-shaped potential," Journal of Mathematical Chemistry, vol. 48, no. 4, pp. 876-882, 2010.

[13] Y.-F. Cheng and T.-Q. Dai, "Exact solution of the Schrödinger equation for the modified Kratzer potential plus a ring-shaped potential by the Nikiforov-Uvarov method," Physica Scripta. An International Journal for Experimental and Theoretical Physics, vol. 75, no. 3, pp. 274-277, 2007. 
[14] A. N. Ikot, E. Olgar, and H. Hassanbadi, "The analytical solutions of the Schrödinger equation with generalized hulthen plus a new ring shaped like potential," Gazi University Journal of Science, vol. 29, no. 4, pp. 937-946, 2016.

[15] A. N. Ikot, I. O. Akpan, T. M. Abbey, and H. Hassanabadi, "Exact Solutions of Schrödinger Equation with Improved Ring-Shaped Non-Spherical Harmonic Oscillator and Coulomb Potential," Communications in Theoretical Physics, vol. 65, no. 5, pp. 569574, 2016.

[16] A. N. Ikot, T. M. Abbey, E. O. Chukwuocha, and M. C. Onyeaju, "Solutions of the Schrödinger equation for pseudo-Coulomb potential plus a new improved ring-shaped potential in the cosmic string space-time," Canadian Journal of Physics, vol. 94, no. 5, pp. 517-521, 2016.

[17] G. Lévai, "SUSYQM and other symmetries in quantum mechanics," Journal of Physics A: Mathematical and General, vol. 37, no. 43, pp. 10179-10191, 2004.

[18] A. N. Ikot, S. Zarrinkamar, S. Zare, and H. Hassanabadi, "Relativistic Dirac-attractive radial problem with Yukawa-like tensor interaction via SUSYQM," Chinese Journal of Physics, vol. 54, no. 6, pp. 968-977, 2016.

[19] R. Butt, A. Khare, and U. P. Sukhatme, "Supersymmetry, shape invariance, and exactly solvable potentials," American Journal of Physics, vol. 56, pp. 163-168, 1988.

[20] A. D. Alhaidari, "An extended class of $L^{2}$-series solutions of the wave equation," Annals of Physics, vol. 317, no. 1, pp. 152-174, 2005.

[21] H. Bahlouli and A. D. Alhaidari, "Extending the class of solvable potential:III:the hyperbolic single wave," Physica Scripta, vol. 81, 2010, 025008.

[22] A. D. Alhaidari, H. Bahlouli, and I. A. Assi, "Solving Dirac equation using the tridiagonal matrix representation approach," Physics Letters A, vol. 380, no. 18-19, pp. 1577-1581, 2016.

[23] I. A. Assi, H. Bahlouli, and A. D. Alhaidari, "Solvable potentials for the 1D Dirac equation using the tridiagonal matrix representations," in Proceedings of the 5th Saudi International Meeting on Frontiers of Physics, SIMFP 2016, Saudi Arabia, February 2016.

[24] A. F. Nikiforov and V. B. Uvarov, Special Functions of Mathematical Physics, Birkhäauser, Basel, Switzerland, 1988.

[25] A. Ikot, E. Maghsoodi, E. Ibanga, E. Ituen, and H. Hassanabadi, "Bound States of the Dirac Equation for Modified Mobius Square Potential Within the Yukawa-Like Tensor Interaction," Proceedings of the National Academy of Sciences India Section A - Physical Sciences, vol. 86, no. 3, pp. 433-440, 2016.

[26] A. D. Antia, A. N. Ikot, H. Hassanabadi, and E. Maghsoodi, "Bound state solutions of Klein-Gordon equation with Mobius square plus Yukawa potentials," Indian Journal of Physics, vol. 87, no. 11, pp. 1133-1139, 2013.

[27] C. Berkdemir, A. Berkdemir, and R. Sever, "Polynomial Solution of the Schrödinger equation for the generalized Woods-Saxon potential," Physical Review C: Nuclear Physics, vol. 74, no. 3, Article ID 039902, 2006.

[28] F. Yasuk, A. Durmus, and I. Boztosun, "Exact analytical solution to the relativistic Klein-Gordon equation with noncentral equal scalar and vector potentials," Journal of Mathematical Physics, vol. 47, no. 8, Article ID 082302, 082302, 8 pages, 2006.

[29] C. Y. Chen, F. L. Lu, D. S. Sun, Y. You, and S. H. Dong, "Exact solutions to a class of differential and some new mathematical properties for the universal associated-legendre polynomials," Appl.Math.Lett.40(2015)90.
[30] D. S. Sun, Y. You, F. L. Lu, C. Y. Chen, and S. H. Dong, "The quantum characteristics of a class of complicated double ring shaped non-central potential," Physica Scripta, vol. 89, 2014, 045002.

[31] C. Y. Chen, F. L. Lu, D. S. Sun, and S. H. Dong, "Analytical solutions of the double ring shaped Coulomb potential in quantum mechanics," Chinese Physics B, vol. 22, Article ID $100302,2013$.

[32] C. Y. Chen, F. L. Lu, and D. S. Sun, "Relativistic scattering states of Coulomb plus a new ring shaped potential," Communications in Theoretical Physics, vol. 45, p. 889, 2016.

[33] L. Y. Wang, X. Y. Gu, Z. Q. Ma, and S. H. Dong, "Exact solutions to $D$-dimensional Schrödinger equation with a pseudoharmonic oscillator," Foundations of Physics Letters, vol. 15, no. 6, pp. 569-576, 2002.

[34] S. H. Dong, "The realization of dynamic group for the pseudoharmonic oscillator," Appl.Math.Lett, vol. 16, p. 199, 2013.

[35] H. Bateman et al., Higher Transcendental Functions, vol. 2, McGraw-Hill, New York, NY, USA, 1955.

[36] J. D. Louck, "Theory Of Angular Momentum in ND.imensional Space," in Los Alamos Scientific Lab.N.Mex, 1960, No.LA-2451.

[37] I. A. Assi, A. J. Sous, and A. N. Ikot, "Using the AIM for solving the non-relativistic wave equation for a new class of infinite onedimensional well with non-flat bottom," The European Physical Journal Plus, vol. 132, no. 12, article no. 525, 2017.

[38] F. J. Ferreira and F. V. Prudente, "Pekeris approximationanother perspective," Physics Letters A, vol. 377, no. 42, pp. 30273032, 2013.

[39] S. I. Zlatev, Pekeris-type approximation for the l-wave in a PöschlTeller potential, 2013.

[40] N. M. Temme, Special Functions: An Introduction to Classical Functions of Mathematical Physics, John Wiley and Sons, New York, NY, USA, 1996.

[41] W. Magnus, F. Onerhetttinger, and R. P. Soni, Formulas and Theorem for the special Functions of Mathematical Physics, Sprnger, Berlin, 1966.

[42] J. Griffiths David, Introduction to quantum mechanics, Cambridge University Press, 2016.

[43] P. Q. Wang, L. H. Zhang, C. S. Jia, and J. Y. Liu, "Equivalence of the three empirical potential energy models for diatomic molecules," Journal of Molecular Spectroscopy, vol. 274, 5 pages, 2012. 

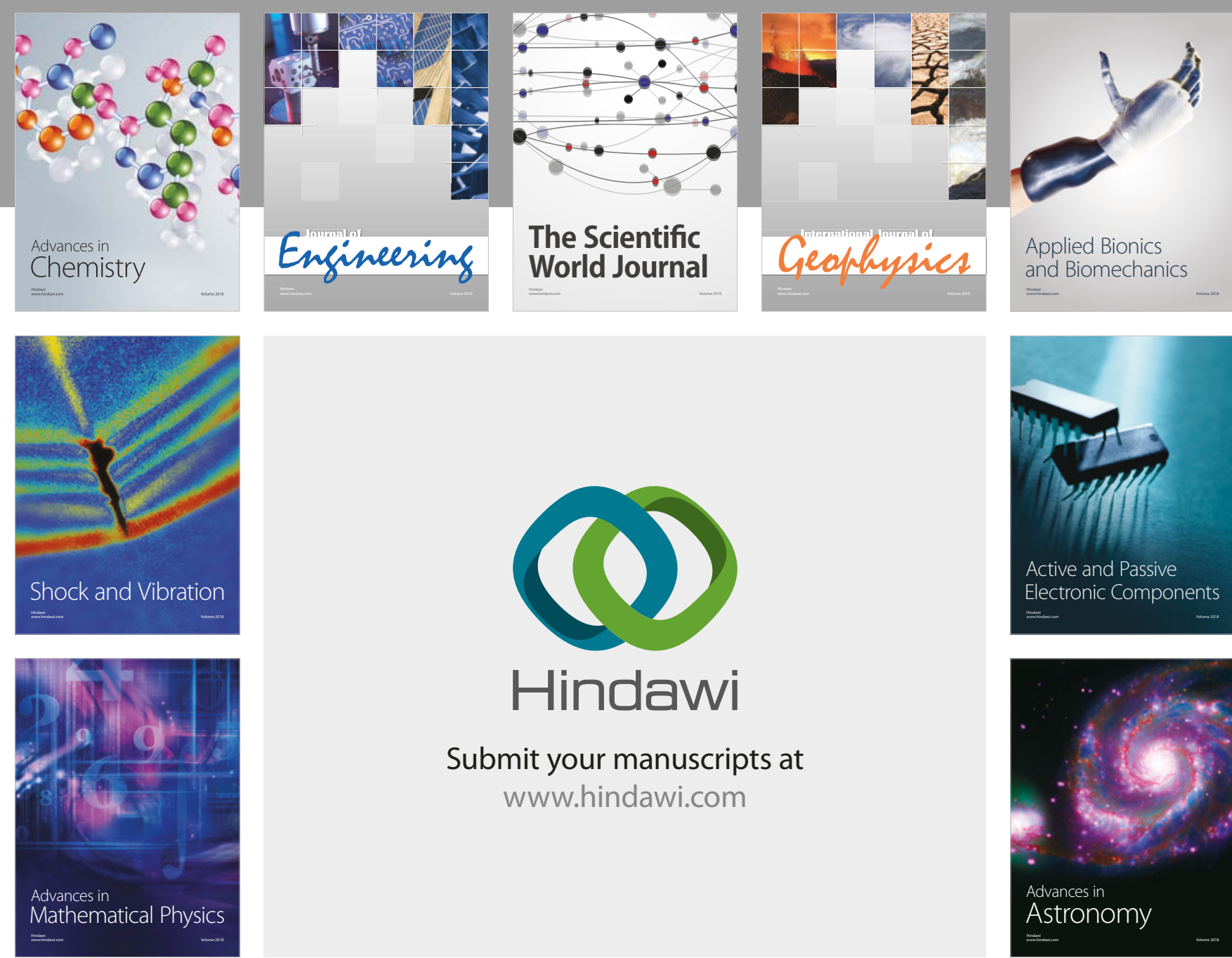

Submit your manuscripts at

www.hindawi.com

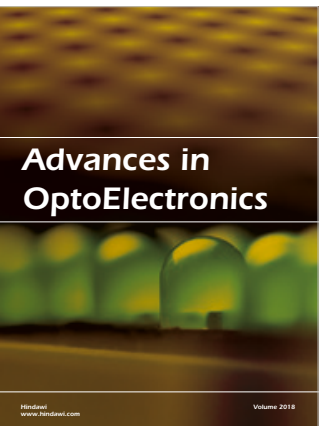

\section{Rotcting Machinery}
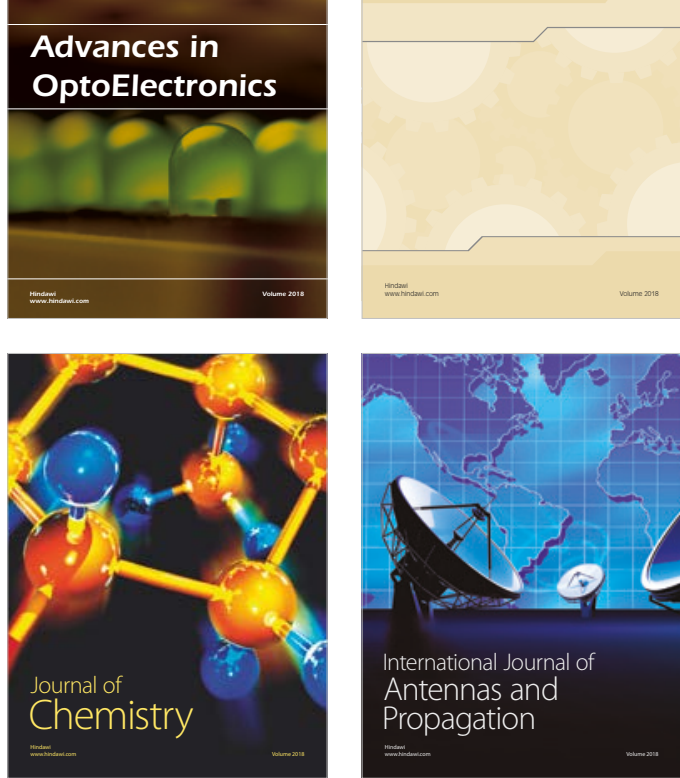

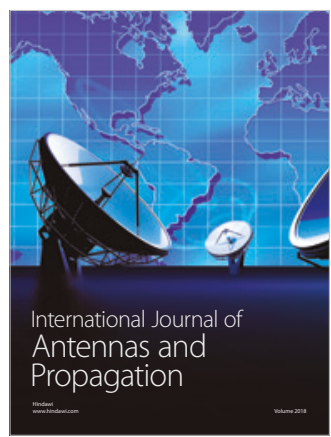

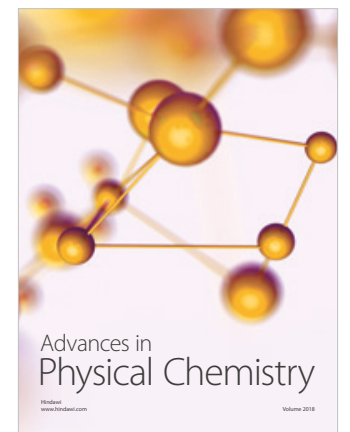

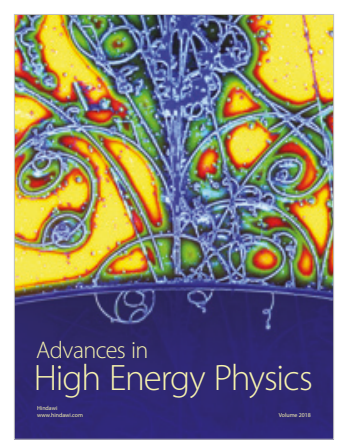

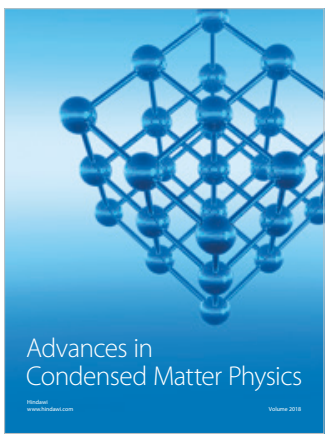

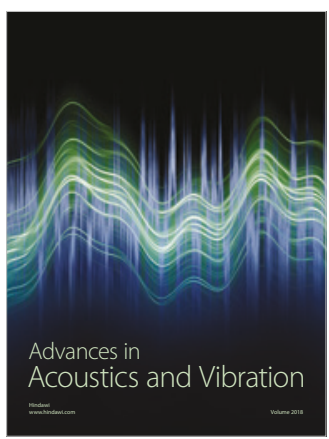

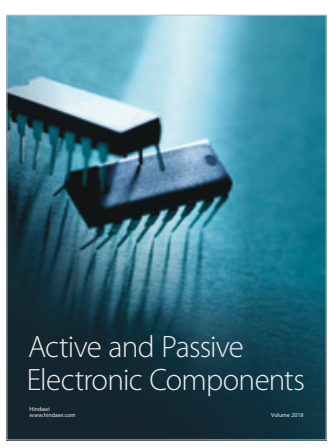
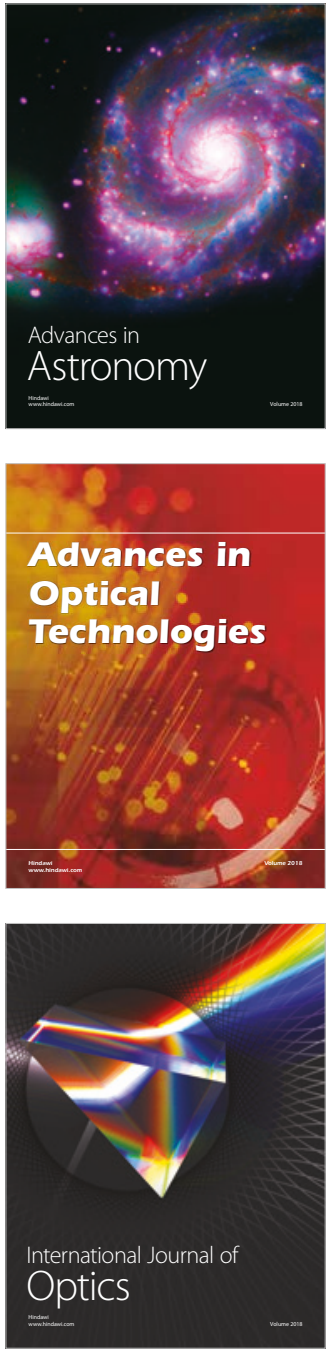